Document downloaded from:

http://hdl.handle.net/10251/99667

This paper must be cited as:

Campos, B.; Cordero Barbero, A.; Torregrosa Sánchez, JR.; Vindel Cañas, P. (2016). Orbits of period two in the family of a multipoint variant of Chebyshev-Halley family. Numerical Algorithms. 73(1):141-156. doi:10.1007/s11075-015-0089-0

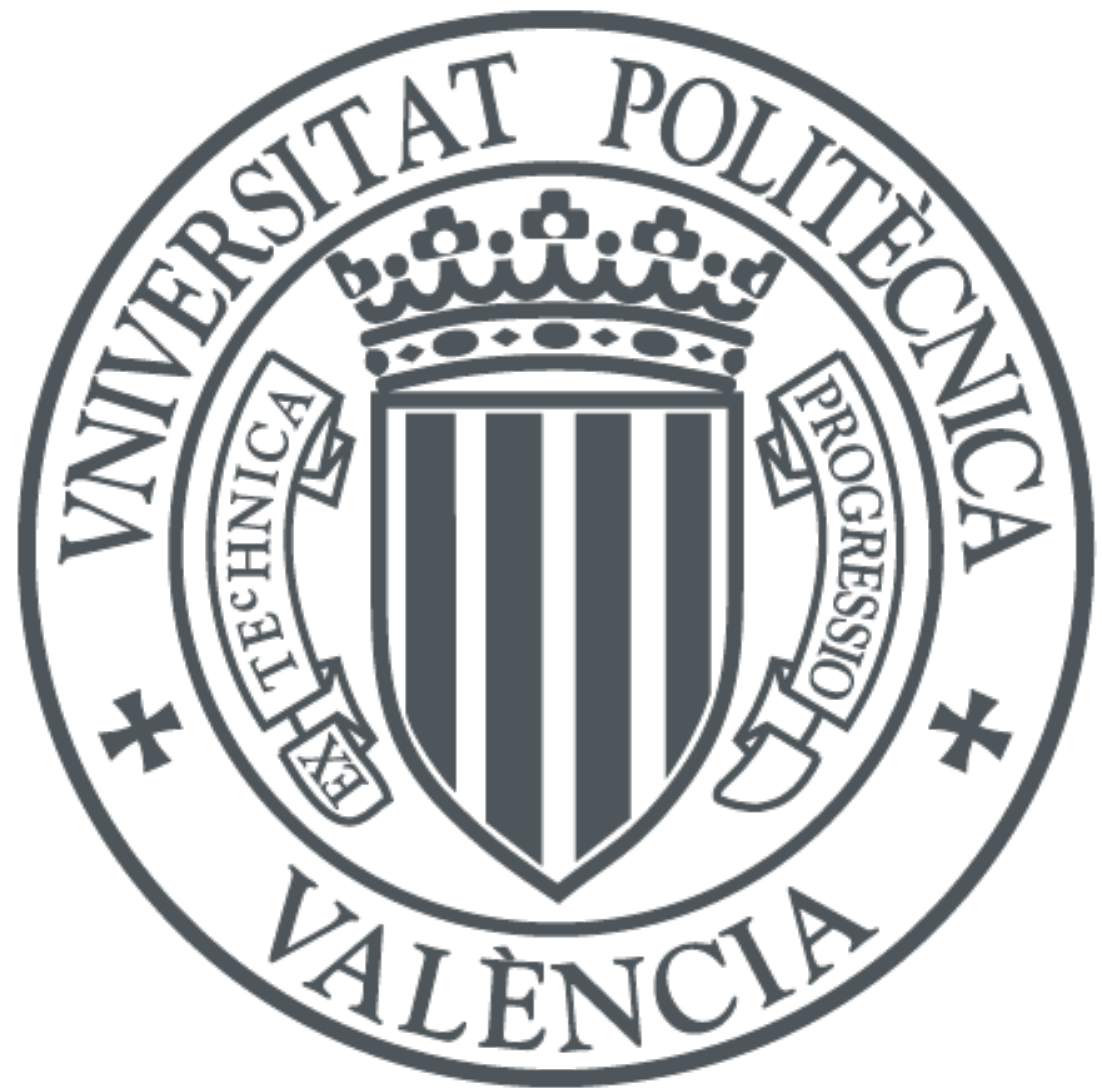

The final publication is available at

http://doi.org/10.1007/s11075-015-0089-0

Copyright Springer-Verlag

Additional Information 


\section{Orbits of period two in the family of a multipoint variant of Chebyshev-Halley family}

Article in Numerical Algorithms · December 2015

DOI: $10.1007 /$ s11075-015-0089-0

\section{CITATION}

1

4 authors:

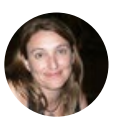

\section{Beatriz Campos}

Universitat Jaume I

37 PUBLICATIONS 66 CITATIONS

SEE PROFILE

\section{Juan R. Torregrosa}

Universitat Politècnica de València
235 PUBLICATIONS 1,911 CITATIONS

SEE PROFILE

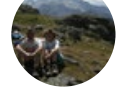

READS

66

\section{Alicia Cordero}

Universitat Politècnica de València

166 PUBLICATIONS 1,627 CITATIONS

SEE PROFILE

P. Vindel

Universitat Jaume I

50 PUBLICATIONS 260 CITATIONS

SEE PROFILE

Some of the authors of this publication are also working on these related projects: 


\title{
Orbits of period two in the family of a multipoint variant of Chebyshev-Halley family
}

\author{
Beatriz Campos · Alicia Cordero · Juan R. \\ Torregrosa - Pura Vindel
}

Received: date / Accepted: date

\begin{abstract}
The study of the dynamical behaviour of the operators defined by iterative methods help us to know more deeply the regions where these methods have a good performance. In this paper, we follow the dynamical study of a multipoint variant of the known Chebyshev-Halley's family, showing the existence of attractive periodic orbits of period 2 for some values of the parameter.
\end{abstract}

Keywords iterative methods $\cdot$ complex dynamics $\cdot$ Chebyshev-Halley's family $\cdot 2$-periodic orbits · 2-bulbs

AMS 2010 Subject Classification codes: 37F10; 65H05

\section{Introduction}

The study of multiple phenomena appearing in different areas of experimental science and technology leads to build a mathematical model.

Mathematical models are of central importance in many scientific contexts. The underlying mathematical nature of the models as the billiard ball model of a gas, the Bohr model of the atom, the Lorenz model of the atmosphere, the Lotka-Volterra model of predator-prey interaction, etc, are cases in point. Mathematical models are one of the principal instruments of modern science.

In many cases, the application of these models to solve a particular problem gives rise to a nonlinear equation or a differential equation or, more often, a system of equations. The problem is that in practice it is very difficult, if not impossible, to find the exact solution of these equations; therefore, it is necessary to resort to numerical approximations by using iterative methods. This means that the output of the method is a sequence of images $\left\{z_{0}, R\left(z_{0}\right), R^{2}\left(z_{0}\right), \ldots, R^{n}\left(z_{0}\right), \ldots\right\}$, where $R$ is a function that represents the fixed-point

This research was partially supported by Ministerio de Economía y Competitividad MTM2014-52016-C022-P.

Beatriz Campos and Pura Vindel

Instituto de Matemáticas y Aplicaciones de Castellón, Universitat Jaume I, Spain

Alicia Cordero and Juan R. Torregrosa

Instituto de Matemática Multidisciplinar, Universitat Politècnica de València, Spain 
operator of the iterative scheme. Therefore, it can be seen as a discrete dynamical system and we can study it from this point of view.

The application of iterative methods on polynomials gives rise to rational functions whose complex dynamics is not well-known, except the case of Newton's method (see, for example, [14]). From the numerical point of view, the dynamical behavior of the rational function associated with an iterative method gives us important information about its stability and reliability. In this sense, Varona in [7] and Amat et al. in [8] described the dynamical behavior of several well-known families of iterative methods. More recently, in [6,9-13], the authors analyze, under the point of view of complex dynamics, the qualitative behavior of different known iterative methods or families, such as King', Chebyshev-Halley's, c-family or damped Newton. When these kind of analysis is made, different pathological numerical behavior, such as periodic orbits, attracting fixed points different from the solution of the problem, etc. A very useful tool to understand the behavior of the different members of the family of methods is the parameter plane, that helps us to select the most stable members of the class.

The natural space for iterating a rational map $R$ is the Riemann sphere $\hat{\mathbf{C}}$. For a given rational map $R$, the sphere splits into two complementary domains: the Fatou set $\mathcal{F}(R)$ where the family of iterates $\left\{R^{n}(z)\right\}_{n \in \mathbf{N}}$ is a normal family, and the Julia set $\mathcal{J}(R)$ where the family of iterates fails to be a normal family. The Fatou set, when it is nonempty, is composed of the set of points whose orbits tend to an attractor (fixed point, periodic orbit, infinity, ...). On the other hand, it is known that the Julia set is a closed, totally invariant, perfect nonempty set, and coincides with the closure of the set of repelling periodic points. The Julia set is the complement of the Fatou set, and it is the locus of chaos; small errors may become arbitrarily large after many iterations. For a deep review on iteration of rational maps see [2]. See [1] for a wide study of complex dynamics.

The first objects to arise in the study of such systems are the so-called fixed points, which are, as the name implies, points of $\hat{\mathbf{C}}$ which are mapped onto themselves by $R$. In complex dynamics, fixed points can be classified by their derivatives into attracting $\left(\left|R^{\prime}\left(z_{0}\right)\right|<1\right)$, superattracting $\left(\left|R^{\prime}\left(z_{0}\right)\right|=0\right)$, repelling $\left(\left|R^{\prime}\left(z_{0}\right)\right|>1\right)$, and neutral points $\left(\left|R^{\prime}\left(z_{0}\right)\right|=1\right)$; these names are indicative of whether nearby points move closer to or further from the fixed point under application of $R$. Besides fixed points, $R$ may have periodic points, which are points that map back to themselves after several iterations of $R$, and pre-periodic points, which are points that eventually map to periodic points.

Given a rational function $R: \hat{\mathbf{C}} \rightarrow \hat{\mathbf{C}}$, where $\hat{\mathbf{C}}$ is the Riemann sphere, the orbit of a point $z_{0} \in \hat{\mathbf{C}}$ is defined as:

$$
\left\{z_{0}, R\left(z_{0}\right), R^{2}\left(z_{0}\right), \ldots, R^{n}\left(z_{0}\right), \ldots\right\}
$$

We are interested in the study of the asymptotic behavior of the orbits depending on the initial condition $z_{0}$, that is, we are going to analyze the phase plane of the map $R$ defined by the different iterative methods. To obtain these phase spaces, the first of all is to classify the starting points from the asymptotic behavior of their orbits.

The basin of attraction of an attractor $\alpha$ is defined as the set of pre-images of any order:

$$
\mathcal{A}(\alpha)=\left\{z_{0} \in \hat{\mathbf{C}}: R^{n}\left(z_{0}\right) \rightarrow \alpha, n \rightarrow \infty\right\} .
$$

The basin of attraction of an attractor needs at least one critical point inside, so, it is important the number of critical points because the critical points are the causative of the instability of numerical methods. A critical point $z_{0}$ is a point where the derivative of rational function vanishes, $R^{\prime}\left(z_{0}\right)=0$. When the iterative method has order of convergence, 
at least, two then the roots of the nonlinear function are superattracting fixed points of the associated operator and so, critical points. However, critical points that are not fixed points of this operator can appear; they are called free critical points.

As we have said, iterative methods are used for finding roots of a nonlinear equation and, from a dynamical point of view, these roots are fixed points of the operator $R$ associated to the method. This paper continues with the dynamical study of a multipoint variant of Chebyshev's method started in [5], where we studied the stability of the fixed points. In Section 2 we summarize some previous results obtained in that paper. In Section 3 we study the existence of periodic orbits and we find three regions in the parameter plane where we can ensure the existence of attractive cycles of period 2 for parameter values in such regions (Propositions 3, 4 and 5). Finally, in Section 4 we obtain the dynamical planes for some values of the parameter where appears a basin of attraction corresponding to a periodic orbit of period 2.

\section{Previous results on the dynamics of a multipoint variant of Chebyshev-Halley family}

The variant of Chebyshev-Halley family is obtained by considering the Newton-like iterative method as a predictor

$$
y_{n}=z_{n}-\alpha \frac{f\left(z_{n}\right)}{f^{\prime}\left(z_{n}\right)}
$$

and modifying the family of Chebyshev's method by using the second-order derivative at $y_{n}$ instead of $z_{n}$ :

$$
z_{n+1}=z_{n}-\frac{f\left(z_{n}\right)}{f^{\prime}\left(z_{n}\right)}-\frac{1}{2} \frac{f\left(z_{n}\right)^{2} f^{\prime}\left(z_{n}\right) f^{\prime \prime}\left(y_{n}\right)}{\left(f^{\prime}\left(z_{n}\right)^{2}-a f\left(z_{n}\right) f^{\prime \prime}\left(y_{n}\right)\right)^{2}} .
$$

As, for $\alpha=\frac{1}{3}$ and $a=\frac{1}{2}$ a fourth-order variant of Chebyshev method is obtained (see [3]), we fix $\alpha=\frac{1}{3}$ and conduct a dynamical study of this family, depending on one parameter $a$.

The fixed point operator corresponding to the family described in (1) is:

$$
G(z, a)=z-\frac{f(z)}{f^{\prime}(z)}-\frac{1}{2} \frac{f(z)^{2} f^{\prime}(z) f^{\prime \prime}(y)}{\left(f^{\prime}(z)^{2}-a f(z) f^{\prime \prime}(y)\right)^{2}},
$$

where

$$
y=z-\frac{1}{3} \frac{f(z)}{f^{\prime}(z)} .
$$

In paper [5] we began the study of the dynamics of this operator applied on quadratic polynomials and using a conjugacy map onto the operator in order to obtain a rational function depending only on the parameter $a$ :

$$
O_{p}(z, a)=\frac{z^{3}(-1+2 a-z)\left(2+(3-2 a) z+z^{2}\right)}{(-1+(2 a-1) z)\left(1+(3-2 a) z+2 z^{2}\right)} .
$$

The fixed points of this operator are the solutions of $O_{p}(z, a)=z$. Solving this equation we obtain $z=0, z=\infty, z=1$ and 


$$
\begin{aligned}
& z_{1,2}(a)=\frac{1}{4}\left(4 a-5+\sqrt{1-8 a} \pm 2 \sqrt{\frac{1}{4}(4 a-5+\sqrt{1-8 a})^{2}-4}\right) \\
& z_{3,4}(a)=\frac{1}{4}\left(4 a-5-\sqrt{1-8 a} \pm 2 \sqrt{\frac{1}{4}(4 a-5-\sqrt{1-8 a})^{2}-4}\right) .
\end{aligned}
$$

A fixed point is attractive or repulsive if the stability function, $\left|O_{p}^{\prime}(z, a)\right|$, is less or greater than one, respectively. As

$$
O_{p}^{\prime}(z, a)=-2 z^{2} \frac{\left(1+(2-2 a) z+z^{2}\right) P(z, a)}{(-1+(2 a-1) z)^{2}\left(1+(3-2 a) z+2 z^{2}\right)^{2}},
$$

where

$$
\begin{aligned}
P(z, a) & =6 a-3+\left(-12+22 a-12 a^{2}\right) z+\left(-18+32 a-24 a^{2}+8 a^{3}\right) z^{2} \\
& +\left(-12+22 a-12 a^{2}\right) z^{3}+(6 a-3) z^{4}
\end{aligned}
$$

we obtain that $z=0$ and $z=\infty$ are always superattractive fixed points, but the stability of the other strange fixed points changes depending on the values of the parameter $a$.

The stability of the fixed points is studied in [5] and the results are summarized in Propositions 1 and 2 .

Proposition 1 For every value $a=x+i y$ of the parameter, the strange fixed point $z=1$ satisfies the following statements :

i) $z=1$ is an attractor inside the curve $C$ defined by:

$$
3 y^{2}=\left(-11+12 x-3 x^{2}+2 \sqrt{-11+12 x-3 x^{2}}\right) .
$$

It is a superattractor for $a=2$.

ii) $z=1$ is a parabolic point for values of the parameter a on the curve $C$, and iii) $z=1$ is a repulsive fixed point for values of a outside the curve $C$.

We also proved that the stability functions of $z_{1}(a)$ and $z_{2}(a)$ coincide; therefore, $z_{1}(a)$ and $z_{2}(a)$ exhibit the same dynamical behavior. The same occurs for $z_{3}(a)$ and $z_{4}(a)$.

Moreover, these fixed points satisfy the following result:

Proposition 2 The fixed points $z_{1}(a)$ and $z_{2}(a)$ are attractors for $a=x+i y \in D$ such that $D=D_{1} \cup D_{2} \cup D_{3}$, where $D_{1}$ is the disk delimited by the circumference $C_{1}$ :

$$
(x-2)^{2}+(y-1.5)^{2}=\frac{1}{4},
$$

$D_{2}$ is the disk delimited by the circumference $C_{2}$ :

$$
(x-2)^{2}+(y+1.5)^{2}=\frac{1}{4},
$$

and $D_{3}$ is delimited by the cardioid $C_{3}$ :

$$
\begin{aligned}
& x(t)=\frac{1}{8}-\frac{1}{4} \frac{6.9}{6000}+\frac{6.9}{6000}\left(\frac{1}{2} \cos t-\frac{1}{4} \cos 2 t\right), \\
& y(t)=\frac{6.9}{6000}\left(\frac{1}{2} \sin t-\frac{1}{4} \sin 2 t\right), t \in[0,2 \pi] .
\end{aligned}
$$


On the other hand, the dynamical behavior of operator $O_{p}(z, a)$ depends on the values of the parameter $a$. The parameter plane is obtained by iterating one free critical point; each point of the parameter plane is associated with a complex value of $a$, i.e., with an element of family (1). The critical points of this system are the roots of $O_{p}^{\prime}(z, a)=0$. From equation (4), we obtain that the critical points are $z=0, z=\infty, c_{ \pm}=a-1 \pm \sqrt{a^{2}-2 a}$ and the roots of a symmetric fourth degree polynomial $P(z, a)$ given by:

$$
\begin{aligned}
& c_{1,2}(a)=\frac{1}{2}\left(x_{+} \pm \sqrt{x_{+}^{2}-4}\right) \\
& c_{3,4}(a)=\frac{1}{2}\left(x_{-} \pm \sqrt{x_{-}^{2}-4}\right),
\end{aligned}
$$

where

$$
x_{ \pm}=\frac{6-11 a+6 a^{2} \pm a \sqrt{1+36 a-12 a^{2}}}{3(2 a-1)} .
$$

Let us remark that, due to the symmetry of $P(z, a), c_{1}(a)=\frac{1}{c_{2}(a)}$ and $c_{3}(a)=\frac{1}{c_{4}(a)}$.

Then, there are free six critical points, different from the roots of the polynomial, but the parameter planes of inverse critical points coincide, i.e. there are only three independent free critical points and three different parameter planes ([?]).

These parameter planes are built by using the algorithms designed in [6], with MatLab program. The following figures are made by using these algorithms, with $2000 \times 2000$ points, 200 iterations and tolerance $10^{-3}$.

In the study of the parameter planes we can ensure that the critical point $c_{1}(a)$ is in the basin of $z=1$ or $z_{1}(a)$ for those values of the parameter for which $z=1$ or $z_{1}(a)$ are attractive, by overlaying their basins of attraction (see Figure 1). In these figures, red color means that the critical point is into the basins of attraction of $z=0$ or $z=\infty$, whereas that black color indicates that the critical point generates its own dynamics.

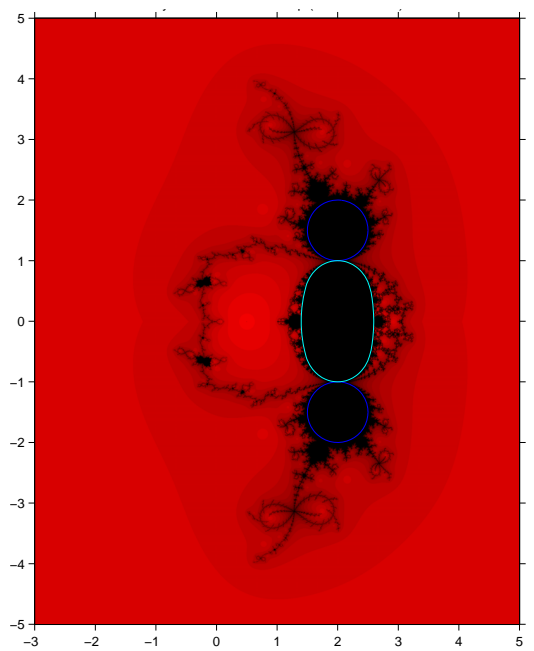

(a) Parameter plane of $c_{1}(a)$ with the curves $C$, $C_{1}$ and $C_{2}$

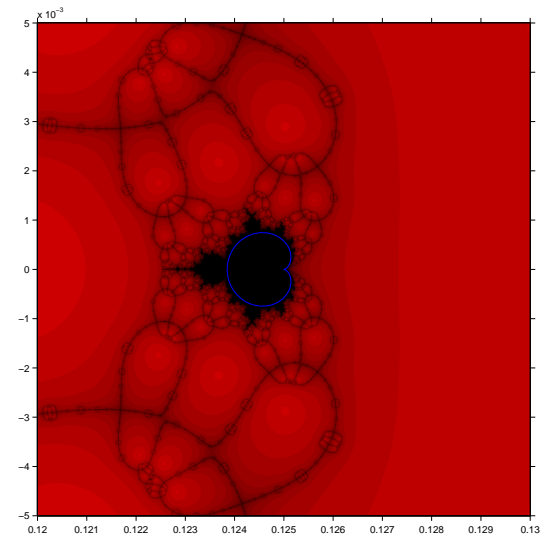

(b) A magnification of the parameter plane of $c_{1}(a)$, with the curve $C_{3}$

Fig. 1: Stability regions of $z=1, z_{1}(a)$ and $z_{2}(a)$ in the parameter plane of $c_{1}(a)$ 
In this paper we study the existence of attractive periodic orbits of period two. We continue exploring the planes of parameters of $c_{1}(a)$ and $c_{3}(a)$. We have found two bulbs in the parameter plane of $c_{1}(a)$ where we can ensure the existence of periodic orbits of period two. We have also found a region in the parameter plane of $c_{3}(a)$ where there also exist 2-periodic orbits.

\section{Regions involving attractive 2-periodic orbits}

The 2-bulbs are regions in the parameter space containing values of the parameter for which the rational operator exhibits attractive orbits of period two.

Cycles of period 2 satisfy the equation:

$$
O_{p}^{2}(z, a)=z .
$$

The relation $O_{p}^{2}(z, a)-z$ can be factorized as:

$$
\begin{aligned}
& (1-z)\left(z^{4}+(5-4 a) z^{3}+\left(4 a^{2}-8 a+8\right) z^{2}+(5-4 a) z+1\right) \\
& \left(z^{6}+(5-4 a) z^{5}+\left(10-12 a+4 a^{2}\right) z^{4}+\left(12-16 a+4 a^{2}\right) z^{3}\right. \\
& \left.+\left(10-12 a+4 a^{2}\right) z^{2}+(5-4 a) z+1\right) P_{24}(z, a)
\end{aligned}
$$

where $P_{24}(z, a)$ is a polynomial of degree 24 in $z$.

The two first factors of $O_{p}^{2}(z, a)-z=0$ give the fixed points; in this paper we show that the third factor gives attractive 2-cycles.

To obtain the solutions of the symmetric 6-degree polynomial, third factor in the previous expression, we follow the process used in [4].

The expressions for these solutions are:

$$
\begin{aligned}
& z_{1,2}(a)=\frac{x_{1} \pm \sqrt{x_{1}^{2}-4}}{2} ; \\
& z_{3,4}(a)=\frac{x_{2} \pm \sqrt{x_{2}^{2}-4}}{2} ; \\
& z_{5,6}(a)=\frac{x_{3} \pm \sqrt{x_{3}^{2}-4}}{2} ;
\end{aligned}
$$

where

$$
\begin{aligned}
x_{1}(a)= & \frac{1}{3}(4 a-5)+\frac{\sqrt[3]{11+24 a+24 a^{2}-16 a^{3}-3 \sqrt{3} \sqrt{-5+48 a-16 a^{2}+96 a^{3}-64 a^{4}}}}{3 \sqrt[3]{2}}+ \\
& \frac{\sqrt[3]{11+24 a+24 a^{2}-16 a^{3}+3 \sqrt{3} \sqrt{-5+48 a-16 a^{2}+96 a^{3}-64 a^{4}}}}{3 \sqrt[3]{2}} ; \\
x_{2}(a)= & \frac{1}{3}(4 a-5)-\frac{(1-i \sqrt{3}) \sqrt[3]{11+24 a+24 a^{2}-16 a^{3}-3 \sqrt{3} \sqrt{-5+48 a-16 a^{2}+96 a^{3}-64 a^{4}}}}{6 \sqrt[3]{2}}- \\
& \frac{(1+i \sqrt{3}) \sqrt[3]{11+24 a+24 a^{2}-16 a^{3}+3 \sqrt{3} \sqrt{-5+48 a-16 a^{2}+96 a^{3}-64 a^{4}}}}{6 \sqrt[3]{2}} ;
\end{aligned}
$$




$$
\begin{aligned}
x_{3}(a)= & \frac{1}{3}(4 a-5)-\frac{(1+i \sqrt{3}) \sqrt[3]{11+24 a+24 a^{2}-16 a^{3}-3 \sqrt{3} \sqrt{-5+48 a-16 a^{2}+96 a^{3}-64 a^{4}}}}{6 \sqrt[3]{2}}- \\
& \frac{(1-i \sqrt{3}) \sqrt[3]{11+24 a+24 a^{2}-16 a^{3}+3 \sqrt{3} \sqrt{-5+48 a-16 a^{2}+96 a^{3}-64 a^{4}}}}{6 \sqrt[3]{2}} .
\end{aligned}
$$

It can be checked that

$$
\begin{aligned}
& O_{p}\left(z_{1}(a), a\right)=z_{2}(a) \text { and } O_{p}\left(z_{2}(a), a\right)=z_{1}(a) \\
& O_{p}\left(z_{3}(a), a\right)=z_{4}(a) \text { and } O_{p}\left(z_{4}(a), a\right)=z_{3}(a) \\
& O_{p}\left(z_{5}(a), a\right)=z_{6}(a) \text { and } O_{p}\left(z_{6}(a), a\right)=z_{5}(a)
\end{aligned}
$$

so, $\left\{z_{1}(a), z_{2}(a)\right\},\left\{z_{3}(a), z_{4}(a)\right\}$ and $\left\{z_{5}(a), z_{6}(a)\right\}$ are periodic orbits of period 2 .

In the following we study for which values of the parameters these 2-cycles are attractive. The coordinates $x$ and $y$ of the parameter plane correspond to the real and imaginary part of the parameter: $a=x+i y$.

A 2-periodic orbit $\left\{z, z^{*}\right\}$ is attractive when $\left|O_{p}^{\prime}(z, a) O_{p}^{\prime}\left(z^{*}, a\right)\right|<1$; if $\left|O_{p}^{\prime}(z, a) O_{p}^{\prime}\left(z^{*}, a\right)\right|>$ 1 the period 2 orbit is repulsive; the values of the parameter $a$ such that $\left|O_{p}^{\prime}(z, a) O_{p}^{\prime}\left(z^{*}, a\right)\right|=$ 1 correspond to values where the orbit changes its stability. In the following results we obtain the regions where $\left\{z_{1}, z_{2}\right\},\left\{z_{3}, z_{4}\right\}$ and $\left\{z_{5}, z_{6}\right\}$ are attractive by finding curves delimiting regions where $\left|O_{p}^{\prime}\left(z_{i}, a\right) O_{p}^{\prime}\left(z_{j}, a\right)\right|<1$.

Proposition 3 The 2-periodic orbit $\left\{z_{1}(a), z_{2}(a)\right\}$ is attractive for $a \in R_{1}$, where $R_{1}$ is a disk delimited by the curve:

$$
\begin{aligned}
& x(t)=1.30932+0.113 \cos t, \\
& y(t)=0.112 \sin t,
\end{aligned}
$$

with $t \in[0,2 \pi]$.

Proof The 2-periodic orbit $\left\{z_{1}(a), z_{2}(a)\right\}$ is attractive for those values of the parameter $a$ such that

$$
S_{12}(a)=\left|O_{p}^{\prime}\left(z_{1}(a), a\right) O_{p}^{\prime}\left(z_{2}(a), a\right)\right|<1 .
$$

We consider a bundle of curves filling the region $R_{1}$ where the outer curve is given by

$$
\begin{aligned}
& x(t)=1.30932+0.113 \cos t, \\
& y(t)=0.112 \sin t,
\end{aligned}
$$

and the rest of curves of the bundle are obtained by decreasing the radii.

As the stability function $S_{12}(a)$ is less than 1 when it is evaluated on this bundle, the 2-cycle $\left\{z_{1}(a), z_{2}(a)\right\}$ is attractive inside the disk $R_{1}$. In Figure 2 we show the bundle of curves and the values of $S_{12}(a)$ evaluated on the bundle.

Note that this outer curve of the bundle corresponding to values of the parameter

$$
a=1.30932+0.113 \cos t+i \cdot 0.112 \sin t, t \in[0,2 \pi]
$$

is an approximation of the set of points such that

$$
\left|O_{p}^{\prime}\left(z_{1}(a), a\right) O_{p}^{\prime}\left(z_{2}(a), a\right)\right|=1 .
$$

Similarly, we obtain the region $R_{2}$ where $\left\{z_{3}(a), z_{4}(a)\right\}$ is attractive. 


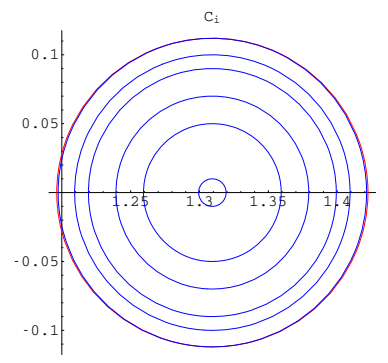

(a) Bundle of curves inside $R_{1}$

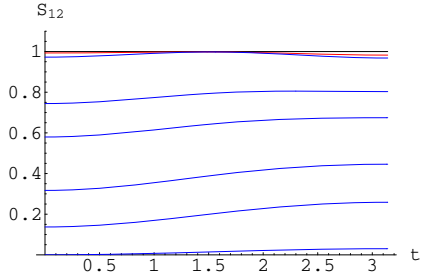

(b) Values of $S_{12}$ on the curves

Fig. 2: Bundle of curves and stability function $S_{12}$ evaluated on the bundle

Proposition 4 The 2-periodic orbit $\left\{z_{3}(a), z_{4}(a)\right\}$ is attractive for a $\in R_{2}$, where $R_{2}$ is a disk delimited by the circumference:

$$
\begin{aligned}
& x(t)=2.66368+0.0825 \cos t, \\
& y(t)=0.0825 \sin t
\end{aligned}
$$

with $t \in[0,2 \pi]$.

Proof The 2-periodic orbit $\left\{z_{3}(a), z_{4}(a)\right\}$ is attractive for those values of the parameter $a$ such that

$$
S_{34}(a)=\left|O_{p}^{\prime}\left(z_{3}(a), a\right) O_{p}^{\prime}\left(z_{4}(a), a\right)\right|<1 .
$$

We consider a bundle of circumferences in the disk $R_{2}$ with the outer curve given by

$$
\begin{aligned}
& x(t)=2.66368+0.0825 \cos t, \\
& y(t)=0.0825 \sin t .
\end{aligned}
$$

The rest of circumferences are obtained by decreasing the radii.

The stability function satisfies $S_{34}(a)<1$ evaluated on each circumference of the bundle; then, the 2-cycle $\left\{z_{3}(a), z_{4}(a)\right\}$ is attractive inside the disk $R_{2}$. In Figure 3 we show the bundle of curves and the values of $S_{34}(a)$ evaluated on it.

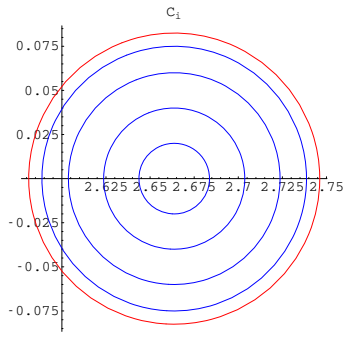

(a) Bundle of curves inside $R_{2}$

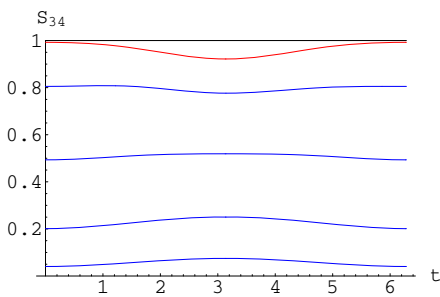

(b) Values of $S_{34}$ on the curves

Fig. 3: Bundle of curves and stability function $S_{34}$ evaluated on the bundle 
In Figure 4 we can observe the regions $R_{1}$ and $R_{2}$ inside the 2-bulbs located on the right and the left of the curve $C$ that delimits the parameter set where the fixed point $z=1$ is attractive.

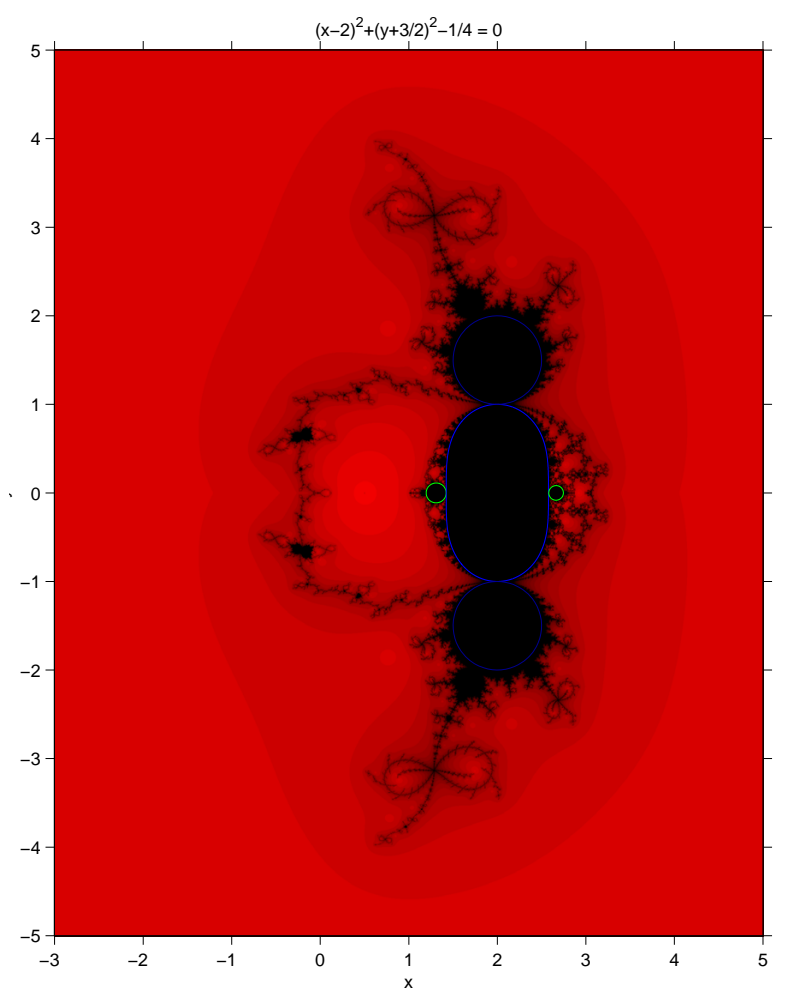

Fig. 4: Parameter plane of $c_{1}(a)$ with the regions $D_{1}, D_{2}, D_{3}, R_{1}$ and $R_{2}$

Finally, the following result shows where the 2-cycle $\left\{z_{5}(a), z_{6}(a)\right\}$ is attractive.

Proposition 5 The 2-periodic orbit $\left\{z_{5}(a), z_{6}(a)\right\}$ is attractive for a $\in R_{3}$, where $R_{3}$ is a region delimited by the cardioid:

$$
\begin{aligned}
& x(t)=1.62-0.117 \cos t(1-\cos t), \\
& y(t)=-0.115 \sin t(1-\cos t),
\end{aligned}
$$

with $t \in[0,2 \pi]$.

Proof The 2-periodic orbit $\left\{z_{5}(a), z_{6}(a)\right\}$ is attractive for those values of the parameter $a$ such that

$$
S_{56}(a)=\left|O_{p}^{\prime}\left(z_{5}(a), a\right) O_{p}^{\prime}\left(z_{6}(a), a\right)\right|<1 .
$$

In this case we consider a bundle of cardioids in order to obtain a better approximation of the boundary of this region of stability. The parametric equation of the outer cardioid is:

$$
\begin{aligned}
& x(t)=1.62-0.117 \cos t(1-\cos t), \\
& y(t)=-0.115 \sin t(1-\cos t),
\end{aligned}
$$


and the rest of curves of the bundle are obtained by decreasing their sizes.

As the stability function $S_{56}(a)$ is less than 1 when it is evaluated on this bundle, the 2-cycle $\left\{z_{5}(a), z_{6}(a)\right\}$ is attractive inside the region $R_{3}$. In Figure 5 we show the bundle of curves and the values of $S_{56}(a)$ evaluated on the bundle.

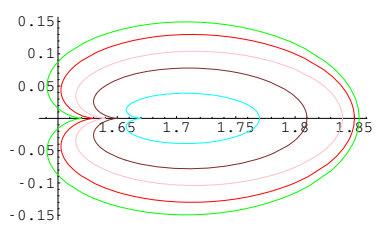

(a) Bundle of curves inside $R_{3}$

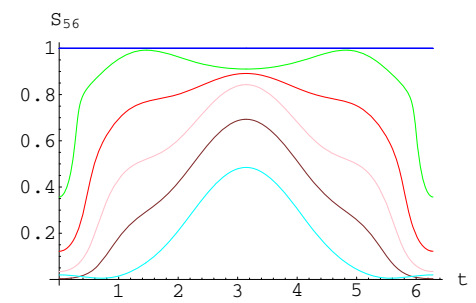

(b) Values of $S_{56}$ on the curves

Fig. 5: Bundle of curves and stability function $S_{56}$ evaluated on the bundle.

So, the region $R_{3}$ corresponds to values of the parameter $a$ where the 2-periodic orbit $\left\{z_{5}(a), z_{6}(a)\right\}$ is attractive.

As we see in Figure 6, this region is located in the parameter plane of the critical point $c_{3}(a)$.

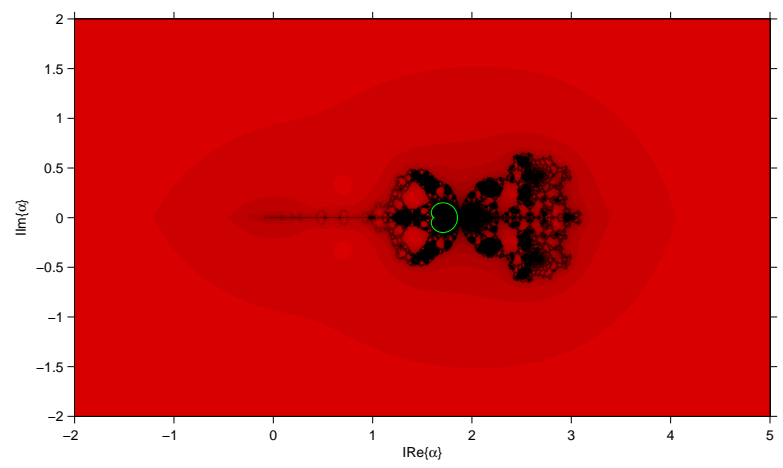

Fig. 6: Parameter plane for $c_{3}(a)$ with the cardioid $R_{3}$.

\section{Dynamical planes with 2-periodic orbits}

In this section we show the dynamical planes for values of the parameter studied in the previous section in order to observe the existence of attractive periodic orbits of period 2.

In Figures 7 and 8 we show the attractive periodic orbits $\left\{z_{1}(a), z_{2}(a)\right\}$ and $\left\{z_{3}(a), z_{4}(a)\right\}$ for values of the parameter in the regions $R_{1}$ and $R_{2}$, respectively. As we have seen in [5], these periodic orbits come from the bifurcation of the fixed point $z=1$. This fixed point is 


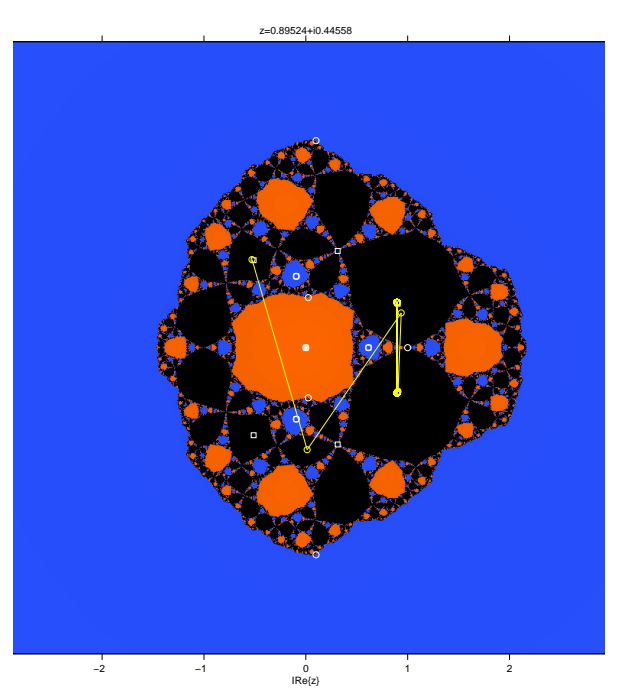

Fig. 7: Dynamical plane for $a=1.3125 \in R_{1}$.

an attractor inside the curve $C$ (see Proposition 1) and it becomes repulsive when entering in region $R_{1}$ at the point $a=2-\frac{\sqrt{3}}{3}$, appearing an attractive double period orbit. The same occurs when $z=1$ enters in region $R_{2}$ at the point $a=2+\frac{\sqrt{3}}{3}$ (see Figure 4).

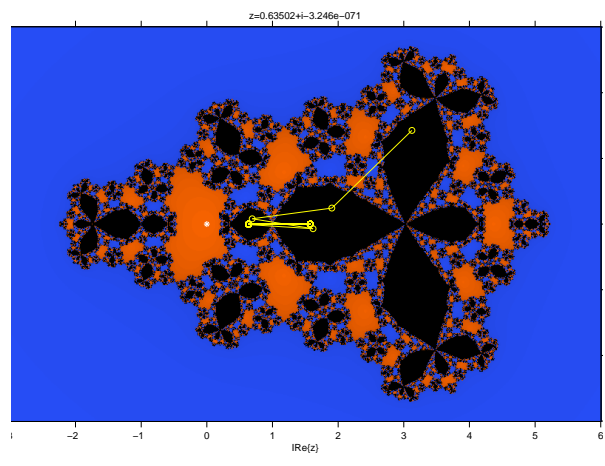

Fig. 8: Dynamical plane for $a=2.6775 \in R_{2}$.

In Figure 9 we can observe the dynamical plane for the parameter $a=1.7175$. In this case, we see the basin of attraction of the orbit of period two $\left\{z_{5}(a), z_{6}(a)\right\}$ (black color) together with the basins of attraction of the fixed points $z=1$ (green color), $z=0$ (orange color) and $z=\infty$ (blue color).

As we have said at the beginning, each attractor needs a critical point inside its basin of attraction. As the critical point $c_{1}(a)$ is in the basin of $z=1$ for this value of the parameter, the basin of attraction of the 2-periodic orbit needs another critical point, that is $c_{3}(a)$. That is the reason why the region $R_{3}$ appears in the parameter plane of $c_{3}(a)$ (see Figure 6). 


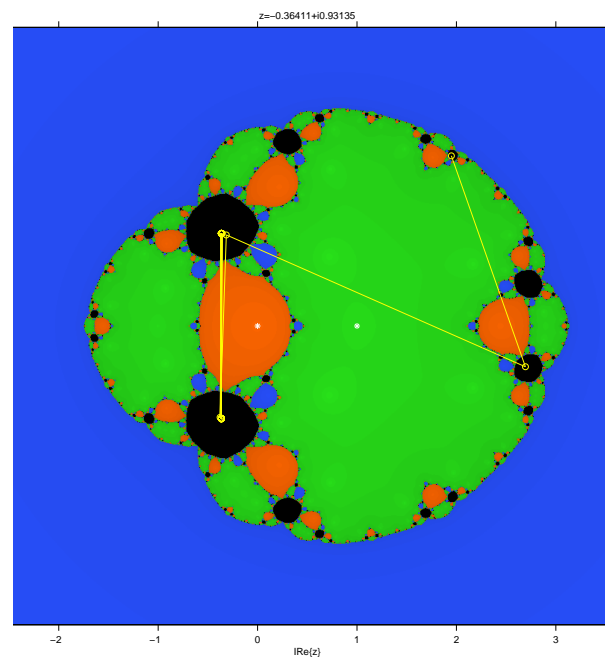

Fig. 9: Dynamical plane for $a=1.7175 \in D_{3}$.

\section{Numerical experiments}

In this section, some numerical examples are given in order to check the behavior of the elements of the analyzed family. We have selected some values of parameter $a$ in stable regions of the parameter plane and also some belonging to unstable regions. For this purpose, we use the following test functions:

- $f_{1}(x)=e^{x}-4 x^{2}, \quad \bar{x}_{1} \approx-0.40777, \quad \bar{x}_{2} \approx 4.306585$,

$-f_{2}(x)=\arctan x, \quad \bar{x}=0$,

- $f_{3}(x)=\left(\sin ^{2} x-\frac{x}{2}\right)^{2}, \quad \bar{x}_{1}=0$ double, $\quad \bar{x}_{2} \approx-1.895494$, double,

- $f_{4}(x)=\sqrt{x^{2}+2 x+5}-2 \sin x-x^{2}+3, \quad \bar{x}_{1} \approx 2.331968 \quad, \bar{x}_{2} \approx-2.573167$,

Numerical computations have been carried out using variable precision arithmetic, with 100 digits, in MATLAB 7.11a. The stopping criterion used is $\left|x_{k+1}-x_{k}\right|<10^{-25}$ or $\left|f\left(x_{k+1}\right)\right|<10^{-25}$. For test function, we calculate the number of iterations, the value of error estimations $\left|x_{k+1}-x_{k}\right|$ and $i n c r=\left|f\left(x_{k+1}\right)\right|$ at the last iteration and the computational order of convergence $A C O C$, approximated by (see [15])

$$
p \approx A C O C=\frac{\ln \left(\left|x_{k+1}-x_{k}\right| /\left|x_{k}-x_{k-1}\right|\right)}{\ln \left(\left|x_{k}-x_{k-1}\right| /\left|x_{k-1}-x_{k-2}\right|\right)} .
$$

The value of $A C O C$ that appears in Table 3 is the last coordinate of vector (8) when the variation between its values is small. In some cases, the approximated order of convergence is not stable and it is shown in the table as '-'.

In Table 1 it can be observed that numerical results confirm the qualitative information obtained in the dynamical study. Parameters belonging to stable regions achieve third- or fourth-order of convergence, meanwhile unstable values of $a$ yield to slower convergence (in terms of number of iterations) or even divergence.

Function $f_{2}(x)$ corresponds to a case of pathological behavior, where divergence appears for all the values of parameter $a$, except for the element of the family with fourth-order of convergence. 


\begin{tabular}{|c|c|c|c|c|c|}
\hline$a$ & root & iter & $\left|x_{k+1}-x_{k}\right|$ & $\left|f\left(x_{k+1}\right)\right|$ & $A C O C$ \\
\hline \hline $1 / 2$ & $\bar{x}_{1}$ & 4 & $3.04 \mathrm{e}-25$ & $5.42 \mathrm{e}-98$ & 3.9890 \\
4 & $\bar{x}_{1}$ & 5 & $6.18 \mathrm{e}-15$ & $1.13 \mathrm{e}-41$ & 3.0072 \\
-2 & $\bar{x}_{1}$ & 5 & $2.7 \mathrm{e}-16$ & $6.73 \mathrm{e}-46$ & 2.9906 \\
2 & $\bar{x}_{1}$ & & NaN & NaN & \\
2.6 & $\bar{x}_{2}$ & 131 & $1.19 \mathrm{e}-16$ & $3.95 \mathrm{e}-46$ & - \\
1.7175 & $\bar{x}_{2}$ & 7 & $5.62 \mathrm{e}-10$ & $2.38 \mathrm{e}-26$ & 3.0050 \\
\hline \hline
\end{tabular}

Table 1: Numerical results for $f_{1}(x)$ and $x_{0}=-1$

\begin{tabular}{|c|c|c|c|c|c|}
\hline$a$ & root & iter & $\left|x_{k+1}-x_{k}\right|$ & $\left|f\left(x_{k+1}\right)\right|$ & ACOC \\
\hline \hline $1 / 2$ & $\bar{x}_{1}$ & 5 & $7.06 \mathrm{e}-22$ & $2.59 \mathrm{e}-108$ & 5.1583 \\
4 & & $>1000$ & & & \\
-2 & & $>1000$ & & & \\
2 & & $>1000$ & & & \\
2.6 & & $>1000$ & & & \\
1.7175 & & $>1000$ & & & \\
\hline \hline
\end{tabular}

Table 2: Numerical results for $f_{2}(x)$ and $x_{0}=1.5$

\begin{tabular}{|c|c|c|c|c|c|}
\hline$a$ & root & iter & $\left|x_{k+1}-x_{k}\right|$ & $\left|f\left(x_{k+1}\right)\right|$ & $A C O C$ \\
\hline \hline $1 / 2$ & $\bar{x}_{1}$ & 22 & $6.04 \mathrm{e}-13$ & $1.35 \mathrm{e}-26$ & 1.0000 \\
4 & $\bar{x}_{1}$ & 27 & $8.60 \mathrm{e}-13$ & $6.65 \mathrm{e}-26$ & 1.0000 \\
-2 & $\bar{x}_{1}$ & 36 & $5.93 \mathrm{e}-13$ & $6.85 \mathrm{e}-26$ & 1.0000 \\
2 & & $>1000$ & & & \\
2.6 & $\bar{x}_{2}$ & 232 & $7.60 \mathrm{e}-13$ & $8.58 \mathrm{e}-26$ & 1.0000 \\
1.7175 & & $>1000$ & & & \\
\hline \hline
\end{tabular}

Table 3: Numerical results for $f_{3}(x)$ and $x_{0}=0.5$

As in case of the first function, the numerical behavior of the members of the family on $f_{3}$ corresponds to the dynamical information obtained in previous sections. However, as the roots in this case are double, convergence is linear.

\begin{tabular}{|c|c|c|c|c|c|}
\hline$a$ & root & iter & $\left|x_{k+1}-x_{k}\right|$ & $\left|f\left(x_{k+1}\right)\right|$ & $A C O C$ \\
\hline \hline $1 / 2$ & $\bar{x}_{1}$ & 4 & $3.86 \mathrm{e}-20$ & $1.66 \mathrm{e}-81$ & 4.3166 \\
4 & $\bar{x}_{1}$ & 5 & $1.47 \mathrm{e}-10$ & $1.07 \mathrm{e}-30$ & - \\
-2 & $\bar{x}_{1}$ & 5 & $1.26 \mathrm{e}-09$ & $4.81 \mathrm{e}-28$ & 3.1058 \\
2 & $\bar{x}_{1}$ & 8 & $1.70 \mathrm{e}-21$ & $7.08 \mathrm{e}-64$ & - \\
2.6 & $\bar{x}_{1}$ & 4 & $1.09 \mathrm{e}-11$ & $2.61 \mathrm{e}-34$ & 2.7894 \\
1.7175 & $\bar{x}_{2}$ & 18 & $4.00 \mathrm{e}-23$ & $1.00 \mathrm{e}-67$ & - \\
\hline \hline
\end{tabular}

Table 4: Numerical results for $f_{4}(x)$ and $x_{0}=5$ 
Table 4 shows a weaker relationship between dynamical and numerical results. In this case, some 'unstable' values of $a$ show a behavior as stable as that of other methods of the family. Nevertheless, unstable performance remains in other elements of the class.

It can be concluded that, in general, the numerical performance of the members of the family belongs to stable regions of the parameter planes. The computational order of convergence confirms the theoretical results.

\section{Final remarks}

There are regions in the parameter planes where there exist attractors that do not correspond to the roots of the equation, so the corresponding numerical method can fail if the initial conditions are not appropriate. Accordingly,

- A dynamical study of the system help us to understand and control the behavior of the numerical method.

- The study of the behavior of a family of numerical methods in terms of the parameters provides information about which members of the family are more efficient. This statement is confirmed by numerical results.

Acknowledgments: The authors thank to the anonymous referees for their suggestions to improve the readability of the paper.

\section{References}

1. P. Blanchard, Complex Analytic Dynamics on the Riemann Sphere, Bull. of the AMS 11(1) (1984) 85141.

2. A. F. Beardon, Iteration of rational functions, Graduate Texts in Mathematics. Springer-Verlag New York, 1991.

3. R Behl and V. Kanwar Variants of Chebyshev's method with optimal order of convergence, Tamsui Oxf. J. Inf. Math. Sci. 29 (1) (2013) 39-53.

4. B. Campos, A, Cordero, A. Magreñan, J.R. Torregrosa and P. Vindel, Study of a bi-parametric family of iterative methods, Abstract and Applied Analysis. Volume 2014. Art. ID 141643, 12 pp.

5. B. Campos, A. Cordero, J.R. Torregrosa and P. Vindel, Bifurcations in the dynamics of a variant of Chebyshev method. Proceedings of the 15th International Conference on Computational and Mathematical Methods in Science and Engineering CMMSE 2015, ISBN 978-84-617-2230-3, (2015) 291-299.

6. F. Chicharro, A. Cordero and J.R. Torregrosa, Drawing dynamical and parameter planes of iterative families and methods, The Scientific World Journal Volume 2013 Article ID 780153

7. J.L. Varona, Graphic and numerical comparison between iterative methods, Math. Intelligencer, 24, 3746, (2002)

8. S. Amat, S. Busquier, S. Plaza, Review of some iterative root-finding methods from a dynamical point of view, Sci. Ser. A: Math. Sci., 10, 3-35, (2004)

9. A. Cordero, J. García-Maimó, J.R. Torregrosa, M.P. Vassileva and P. Vindel, Chaos in King's iterative family, Appl. Math. Lett., 26, 842-848, (2013)

10. A. Cordero, J.R. Torregrosa and P. Vindel, Dynamics of a family of Chebyshev-Halley type method, Appl. Math. and Comput., 219, 8568-8583, (2013)

11. J.M. Gutiérrez, M.A. Hernández and N. Romero, Dynamics of a new family of iterative processes for quadratic polynomials, J. of Comput. and Appl. Math., 233, 2688-2695, (2010)

12. B. Neta, C. Chun, M. Scott, Basins of attraction for optimal eighth order methods to find simple roots of nonlinear equation, App. Math. Comput., 227, 567-592, (2014)

13. M. Scott, B. Neta, C. Chun, Basin attractors for various methods, Appl. Math. and Comput., 218, 25842599, (2011)

14. P. Blanchard, The Dynamics of Newton's Method, Proc. of Symp. in Appl. Math., 49, 139-154, (1994)

15. A. Cordero and J.R. Torregrosa, Variants of Newton's method using fifth-order quadrature formulas, Appl. Math. and Comput., 190 686-698, (2007) 